\title{
Proper orthogonal decomposition versus Krylov subspace methods in reduced-order energy-converter models
}

\author{
MD Rokibul Hasan, Ruth V. Sabariego \\ EnergyVille, KU Leuven, Belgium
}

\author{
Christophe Geuzaine ${ }^{1}$, Yannick Paquay ${ }^{1,2}$ \\ ${ }^{1}$ ACE, University of Liège, ${ }^{2}$ F.R.S.-FNRS, Belgium
}

\begin{abstract}
In this paper, the proper orthogonal decomposition and the Arnoldi-based Krylov subspace methods are applied to the magnetodynamic finite element analysis of power electronic converters. The performance of these two model order reduction techniques is compared both in frequency and time domain. Moreover, two original, adaptive and automated greedy snapshots selection methods are investigated using either local or global quantities for selecting the snapshots (frequencies or time steps).

Index Terms-Reduced-order model, proper orthogonal decomposition, Krylov subspace methods, finite elements, eddy currents.
\end{abstract}

\section{INTRODUCTION}

Power converters, e.g. transformers, may be subjected to significant eddy current effects that can alter their performance. The accurate consideration of these dynamic effects is thus a major concern from early stages of the electromagnetic design [1], [2]. The finite element (FE) method is widely used for numerically modelling these electromagnetic phenomena [3]. It can be extremely expensive though, particularly when accounting for non-linearities (ferromagnetic materials, fast switching) and modern pulsed-width modulated supplies (PMW) working at ever-increasing frequencies. The discretisation in space and time can thus yield a large system of equation with a prohibitive computational cost (time and storage). Indeed, we need a fine mesh to capture the highfrequency induced currents (concentrated at the surface of the conductors) with also a possibly long transient behaviour.

In the literature the most popular approaches for handling this issue are those based on the extraction of physical parameters such as inductances, flux linkages, reluctances,... [4]. The reluctance identification allows replacing a FE model by a magnetic circuit model (MEC) with relatively high accuracy and low computational cost [5]. Indeed, a MEC allows accounting for harmonics due to slotting, saturation, eccentricity and faults, in flux linkages, currents and torque with easiness and precision [6]. Also the hysteresis behaviour can be considered [7]. The FE-based parameter extraction combines the feature of the circuit models and the full FE model. The parameters (inductances or flux linkages) are obtained from the solutions of a series of FE computations,

\footnotetext{
Work supported by Belgian Science Policy (IAP P7/02).
}

978-1-4673-8463-6/16/\$31.00 (C) 2016 European Union covering the operating range of the power converter. They are stored in tables then utilized by table look up according to the actual operating condition of device [4]. A survey on the numerical techniques particular for modelling transformers can be found in [2].

Model order reduction (MOR) techniques have become a feasible and efficient alternative in numerical electromagnetics [8], [9]. MOR methods reduce the original matrix system by projecting the original basis to a reduced subspace. Snapshot techniques are most often used to build the projection operator [9].

This paper aims at modelling a magneto-quasi-static (eddy current) problem by means of two reduced order techniques, namely the Proper Orthogonal Decomposition (POD) [10] and Arnoldi based Krylov Subspace (AKS) methods [11]. While the POD is applicable in both the time and frequency domain, the AKS is only valid in the frequency domain. The comparison in the time domain is still possible by applying the harmonic formalism.

The performance of POD and AKS has already been confronted in [12], however, herein snapshots are chosen without greedy algorithms. Indeed in the frequency domain, a uniform snapshot/frequency distribution is used and in the time domain, the bases are generated from a given number of time steps, fixed a priori and increased till desired accuracy is achieved in a trial and error approach. In this paper, we extend our frequency-domain algorithm [13] to the time domain and propose an original and efficient greedy algorithm in time domain. Furthermore, we compare both methods in time with projector operators generated by greedy approaches either in the frequency or time domain.

\section{Magnetodynamic MOdel}

Let us consider a bounded domain $\Omega=\Omega_{c} \cup \Omega_{c}^{C} \in \mathbb{R}^{3}$ with boundary $\Gamma$. The conducting and non-conducting part of $\Omega$ are denoted by $\Omega_{c}$ and $\Omega_{c}^{C}$, respectively. The classical (modified) magnetic-vector-potential $(a-)$ magnetodynamic formulation (weak form of Ampère's law) reads: find $a$, such that

$\left(\nu \operatorname{curl} a, \operatorname{curl} a^{\prime}\right)_{\Omega}+\left(\sigma \partial_{t} a, a^{\prime}\right)_{\Omega_{c}}+\left\langle\hat{n} \times h, a^{\prime}\right\rangle_{\Gamma}=\left(j_{s}, a^{\prime}\right)_{\Omega_{s}}$

holds for all test functions $a^{\prime}$ in a suitable function space; $b=\operatorname{curl} a$ is the magnetic flux density (or induction); $j_{s}$ is 
a prescribed current density; $\hat{n}$ is the outward unit normal vector on $\Gamma ;(\cdot, \cdot)_{\Omega}$ and $\langle\cdot, \cdot\rangle_{\Omega}$ denote a volume integral in $\Omega$ and a surface integral on $\Gamma$ of the scalar product of their arguments. For the sake of simplicity, the media is assumed linear and isotropic, with magnetic constitutive law $h=\nu b$ (magnetic field $h$, reluctivity $\nu$ ) and electric constitutive law $e=\sigma j=-\sigma \partial_{t} a$ (electric field $e$, induced current density $j$, conductivity $\sigma$ ).
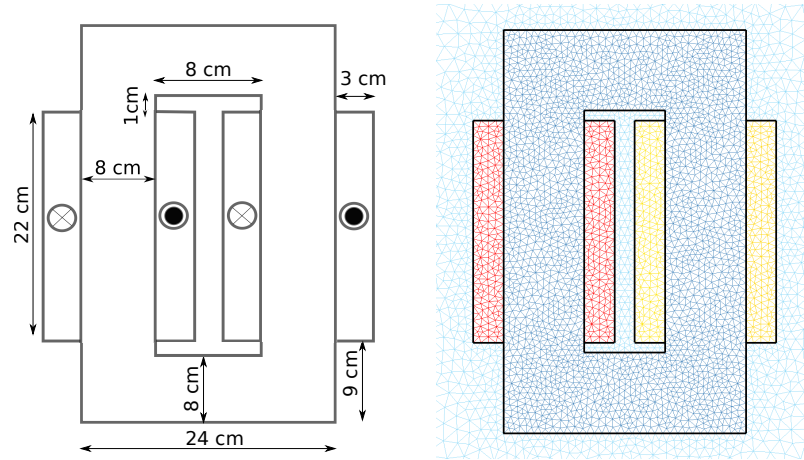

Fig. 1. Geometry (left) and mesh (right) of the single-phase transformer considered for validation

The FE discretisation of (1) with $N$ edge basis functions for $a$ and $a^{\prime}$ (Galerkin approach), leads to the following system of first-order differential equations:

$$
A \partial_{t} x(t)+B x(t)=b(t),
$$

where $x(t)$ is the time-dependent column vector of $N$ unknowns, $A, B$ are $N \times N$ matrices of coefficients and $b(t)$ is the source column vector (right hand side).

Furthermore, the system (2) is discretized in time by means of the so-called $\theta$-scheme, which amounts to implicit or backward Euler with $\theta=1$, the adopted scheme. A system of algebraic equations is obtained for each time-step from $t_{k-1}$ to $t_{k}=t_{k-1}+\Delta t$.

Taking this into account, discretized system (2) in time reads:

$$
\left[A_{\Delta t}+B\right] x_{k}=A_{\Delta t} x_{k-1}+b_{k}
$$

with $A_{\Delta t}=\frac{A}{\Delta t}, x_{k}=x\left(t_{k}\right)$ the solution at instant $t_{k}, x_{k-1}=$ $x\left(t_{k-1}\right)$ the solution at instant $t_{k-1}, b_{k}$ the right hand side at $t_{k}$ and $\Delta t$ the time step.

Assuming a sinusoidal source (frequency $f$, pulsation $\omega=$ $2 \pi f$ ), we can apply the complex formalism (complex in bold) and rewrite (2) in frequency domain as

$$
[\imath \omega A+B] \boldsymbol{x}=\boldsymbol{b},
$$

where the dependence of $\boldsymbol{x}$ and $\boldsymbol{b}$ with the frequency $(f, \omega)$ is omitted for the sake of simplicity.

\section{MOdEL ORder REDUCTION}

Model order reduction techniques aim at reducing the matrix system in the time domain (3) or in the frequency domain (4) [8]. The solution vector $x(N \times 1)$ is then approximated by a vector $x^{r}(M \times 1)$ in a reduced basis, $M \ll N$,

$$
x \approx \Psi x^{r},
$$

with $\Psi$ an orthonormal projection operator; respectively $\boldsymbol{x}, \boldsymbol{x}^{r}$ and $\boldsymbol{\Psi}$ in the frequency domain. Therefore, the reduced-order systems of (3) and (4) become, respectively,

$$
\begin{aligned}
{\left[A_{\Delta t}^{r}+B^{r}\right] x_{k}^{r} } & =A_{\Delta t}^{r} x_{k-1}^{r}+b_{k}^{r}, \\
{\left[\boldsymbol{\imath} \omega A^{r}+B^{r}\right] \boldsymbol{x}^{r} } & =\boldsymbol{b}^{r},
\end{aligned}
$$

with $A_{\Delta t}^{r}=\Psi^{T} A_{\Delta t} \Psi, B^{r}=\Psi^{T} B \Psi$ and $b^{r}=\Psi^{T} b$ in (6) and analogously, $A^{r}=\boldsymbol{\Psi}^{T} A \boldsymbol{\Psi}, \boldsymbol{b}^{r}=\boldsymbol{\Psi}^{T} \boldsymbol{b}$ in (7).

In this paper the projection operators $\Psi, \Psi$ are constructed either using the POD or the AKS method.

\section{A. Proper Orthogonal Decomposition (POD)}

In the POD method, the $\Psi(\Psi)$ operator is constructed based on the snapshots techniques [9], i.e. generated from the time-domain full solution $x(\boldsymbol{x})$. Note that quantities between parenthesis correspond to the frequency domain case.

Let us consider the snapshot matrix $S=\left[x_{1}, x_{2}, \ldots\right](\mathcal{S}=$ $\left.\left[x_{1}, x_{2}, \ldots\right]\right)$ from the set of solution $x(x)$ for the selected number of time steps (frequencies).

Applying the singular value decomposition (SVD) to this snapshot matrix $\mathcal{S}$ as,

$$
\mathcal{S}=\mathcal{U} \Sigma \mathcal{V}^{T}, \quad\left(\mathcal{S}=\mathcal{U} \Sigma \mathcal{V}^{T}\right)
$$

In the general case $\Psi=\mathcal{U}^{r}\left(\Psi=\mathcal{U}^{r}\right)$ where $\mathcal{U}^{r}\left(\mathcal{U}^{r}\right)$ corresponds to the truncation ( $r$ first columns) of $\mathcal{U}(\mathcal{U})$. In this paper, as the number of time steps (frequencies) to compute the $\Psi(\Psi)$ operator must be as small as possible, it is directly obtained by taking $\Psi=\mathcal{U}(\Psi=\mathcal{U})$ without truncation.

\section{B. Arnoldi-based Krylov Subspace (AKS) method}

The AKS approach can only be applied in the frequency domain. The construction of $\boldsymbol{\Psi}$ is based on the Krylov subspace $\mathcal{K}_{n}(\alpha, \gamma)=\operatorname{span}\left\{\gamma, \alpha^{1} \gamma, \alpha^{2} \gamma, \cdot \alpha^{n-1} \gamma\right\}$ and on the transfer function of (4). After applying the Laplace transformation, it reads

$$
H(s)=(A s+B)^{-1} \boldsymbol{b},
$$

that is further approximated with a Padé expansion around the expansion point, $\boldsymbol{s}_{\exp }[12]$.

It reads

$$
H(s)=\sum_{j} H_{j}\left(s-s_{e x p}\right)^{j}
$$

with $H_{j}=\left(-\left(A \boldsymbol{s}_{\text {exp }}+B\right)^{-1} A\right)^{j}\left(A \boldsymbol{s}_{\text {exp }}+B\right)^{-1} \boldsymbol{b}$ with $\alpha=$ $-\left(A \boldsymbol{s}_{\text {exp }}+B\right)^{-1} A, \gamma=\left(A \boldsymbol{s}_{\text {exp }}+B\right)^{-1} \boldsymbol{b}$ and $j=0,1, \ldots, n-$ 1. The set of the vectors $H_{j}$ constructs Krylov subspaces as $H_{0}=\alpha, H_{1}=\alpha^{1} \gamma$ and so on. The Arnoldi algorithm is used for generating the projection basis $\boldsymbol{\Psi}$ from the Krylov subspace [14]. Hence, $\boldsymbol{\Psi}$ is built from the orthogonal basis of $\mathcal{K}_{n}(\alpha, \gamma)$. Notice that, in this paper, we consider the first two moments for each expansion point in Arnoldi. Indeed, with only one moment for the expansion point the approach is theoretically analogous to the POD in frequency [11]. Since 
the AKS method uses two moments, it doubles the reduced size $M$ (for an identical number of selected frequencies) with regard to the POD.

\section{Switching bases between frequency and time domain}

Because we generate the projection operators in the frequency domain for future use in time domain, one may wonder if the projection operator can be directly interchanged from frequency to time domain (and vice-versa) since it is complex. By applying the harmonic formalism $x_{t}=\|\boldsymbol{x}\| e^{\boldsymbol{\imath} \omega t}$, (5) becomes

$$
\begin{aligned}
x_{t} & \approx \Psi x_{t}^{r} \\
& =\Psi \Re\left\{\left\|\boldsymbol{x}^{r}\right\| e^{\boldsymbol{\imath} \omega t}\right\} \\
& =\Re\left\{\Psi\left\|\boldsymbol{x}^{r}\right\| e^{\boldsymbol{\imath} \omega t}\right\}
\end{aligned}
$$

and $x_{t} \approx \Re\left\{\boldsymbol{\Psi}\left\|\boldsymbol{x}^{r}\right\| e^{\boldsymbol{\imath} \omega t}\right\}$ [11]. By identification, the two bases $\Psi, \Psi$ (in time and frequency domain, respectively) can be thus switched.

\section{GREEDY Algorithms}

Being able to approximate the solution vector $x, \boldsymbol{x}$ (in time or frequency domain respectively) in a small dimensional subspace $x^{r}, \boldsymbol{x}^{r}$ does not mean that a pertinent discrete scheme is easy to find. Such a discrete space comprises solutions of (3), (4) for well chosen parameters, e.g. $M$ instants or frequencies. A greedy algorithm provides such an opportunity [15], [16].

\section{A. Time Domain}

From (3), the solution $x_{k}$ depends on $x_{k-1}$ and prohibits parallel computations to efficiently build the reduced basis (RB). Indeed, in [12], [17], [18], the first $K_{s}(<K)$ temporal solutions are fully computed and directly used to generate the POD basis for future use (i.e. next time steps). Unfortunately, this approach lacks the arbitrary choice of $K_{s}$. We propose a greedy algorithm that automatically detects the minimum required time steps $K_{s}$ to build a POD reduced basis. Note that since this reduced basis can be truncated, $M$ may differ from $K_{s}$.

Given a number of trials (or predictions) $n b$ and a tolerance $\tau$, the proposed algorithm 1 solves the high fidelity model and the candidate reduced model (building the RB online) for each time step till the error of the observed quantity between the reference and the reduced results is $n b$ times lower than the tolerance $\tau$. When a single trial fails the error bound limitation, the counter is reset and a new RB must be computed using all the previously stored snapshots. The aforementioned quantity can be local (LQ), e.g. the full unknown vector describing the solution at every point of the mesh, e.g. the magnetic flux density, the current density; or global (GQ), e.g. the losses. The higher the number of trials $n b$, the more predictive the candidate reduced model becomes.

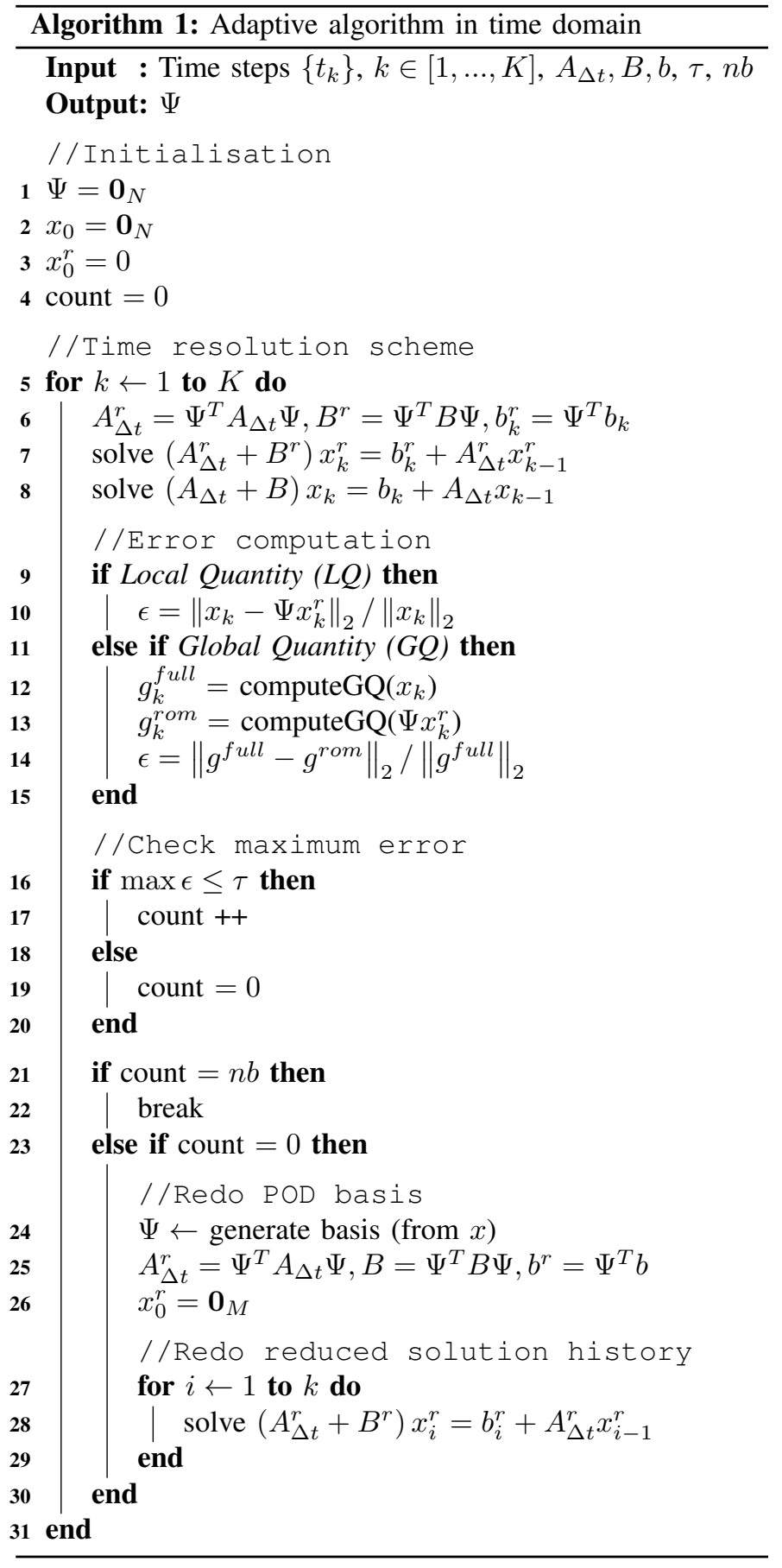

\section{B. Frequency Domain}

Contrary to the time domain (3), the frequency domain solutions (4) are independent for different frequencies allowing a greedy approach to efficiently build the RB by computing the specific requested responses. In [13], the authors proposed an original greedy selection method to find the minimum number of snapshots $M$ within a prescribed tolerance $\tau$ for the error $\epsilon$ on a quantity of interest. It solves the original system for well chosen frequencies, increases the reduced basis, and stops when the observed quantity has converged (e.g. $L^{2}$ - 
norm of the error between the previous and current results). During the execution, the next selected snapshot is chosen at the frequency with the highest variation with regard to the previous result-referring to the numerical convergence of the observed quantity. Analogous to the time greedy approach, the observed quantity can be local or global.

\section{ApPLICATION EXAMPLE}

As test case we consider the single-phase power transformer depicted in Fig. 1. It consists of an iron core and two coils wound around. The coils are made of copper (conductivity $\sigma_{C u}=5.7710^{7} \mathrm{~S} / \mathrm{m}$, non-magnetic) with 358 and 206 turns on the primary and secondary side, respectively. The secondary winding is an open-circuit. The core is assumed linear and isotropic with relative permeability $\mu_{r}=1000$ and conductivity $\sigma_{\text {core }}=3.7210^{3} \mathrm{~S} / \mathrm{m}$. The geometry is meshed with 8430 triangles, yielding 4175 unknowns with a classical FE approach. This discretisation is kept constant for all considered cases.

The magnetodynamic problem is solved both in the time and frequency domain. The primary coil is fed with a sinusoidal current, the frequency of which is chosen in the range $f \in$ $[0,100] \mathrm{Hz}$. The time simulation is performed at $f=50 \mathrm{~Hz}$ with 61 time-steps per period over one period and a half.

\section{A. Projection operator $\Psi$ generated in frequency}

The POD and AKS techniques are used to generate the complex projection operator $\Psi$. The obtained reduced basis is then used in the time domain for the computation of global quantities (losses, flux linkage,...) or local quantities (magnetic flux density or induction, current density, ...).

In order to compare, not only the RO methods: POD and AKS, but also the efficient ways of basis generation: uniform snapshot selection [12] and greedy snapshot selection [13], we solve a magnetodynamic problem for model (4) in frequency domain, the basis is then used in the frequency and time domain to compute the global and local quantities of interest. Compared to the uniformly taken frequencies result of [12] (with $f \in[0,100] \mathrm{Hz}$ ) to generate snapshots and projection basis for both POD and AKS methods (Fig. 2), the automated greedy algorithm described in [13] has been used to find the best set of frequencies (Fig. 5).

1) Uniform snapshots selection: In Fig. 2, it can be observed that, the average $L^{2}$-relative error of core losses for the frequency basis used in frequency domain for $M=2,4,6$, POD and $M=4,8,12$, AKS is $10^{-2}, 10^{-4}, 10^{-8}$ for both methods. Using the projector operator $\boldsymbol{\Psi}$ in time domain provides the results depicted in Fig. 3, where the average $L^{2}$ relative error of core losses is below $1 \%$ for $M \geq 6$ using both POD and AKS.

2) Greedy snapshots selection: Concerning the convergence rates of the greedy algorithm for both reduction techniques, the POD decays twice faster than the AKS convergence (see Fig. 4). Be aware that the abscissa is the size of the reduced system and that the AKS method uses two moments,
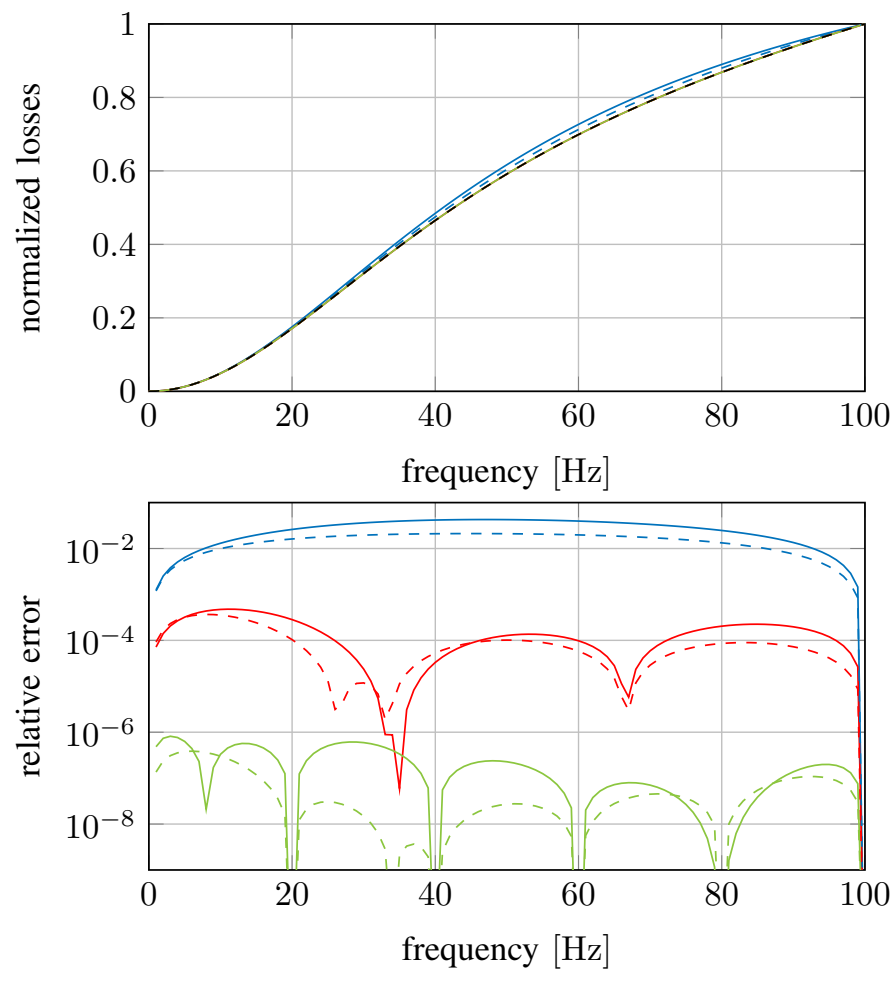

Fig. 2. Uniform frequency algorithm - normalized losses in transformer core with POD and AKS (up) and $L^{2}$-relative error (down). FEM (-- ), POD $\mathrm{M}=2(-), 4(-), 6(-), \mathrm{AKS} \mathrm{M}=4(---), 8(---), 12(--)$
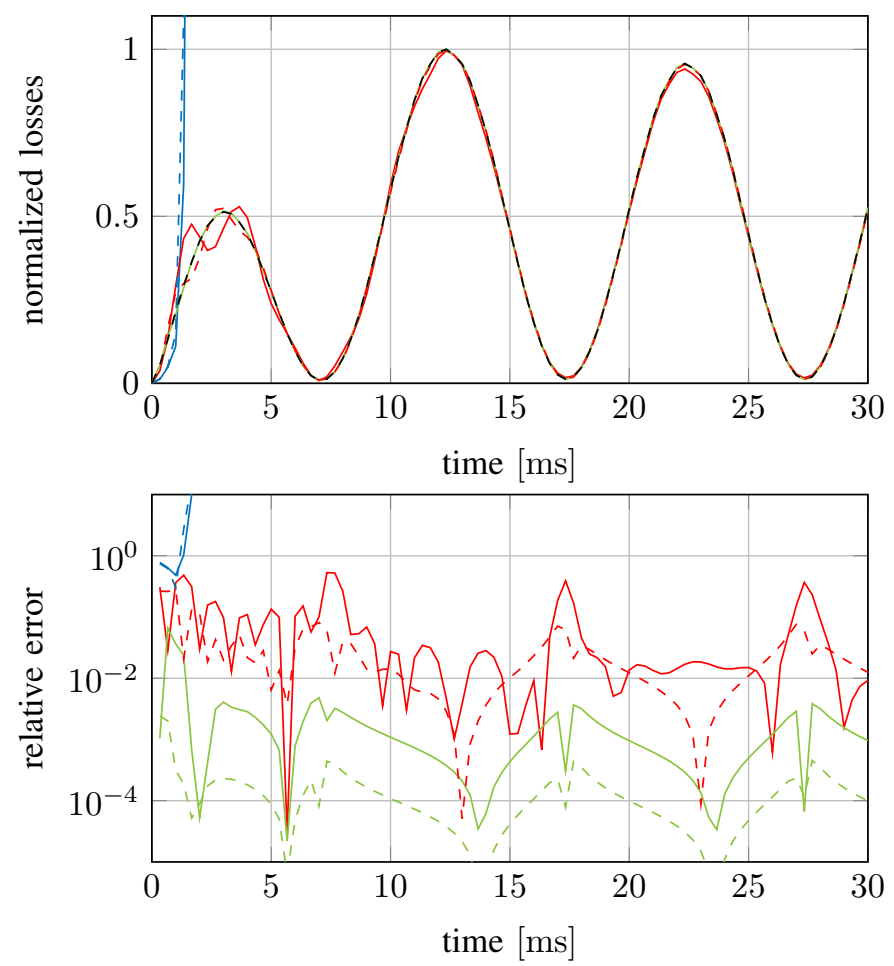

Fig. 3. Uniform frequency algorithm - normalized losses in transformer core with POD and AKS (up) and $L^{2}$-relative error (down). FEM (-- ), POD $\mathrm{M}=2(-), 4(-), 6(-)$, AKS M=4(-- $), 8(---), 12(---)$ 


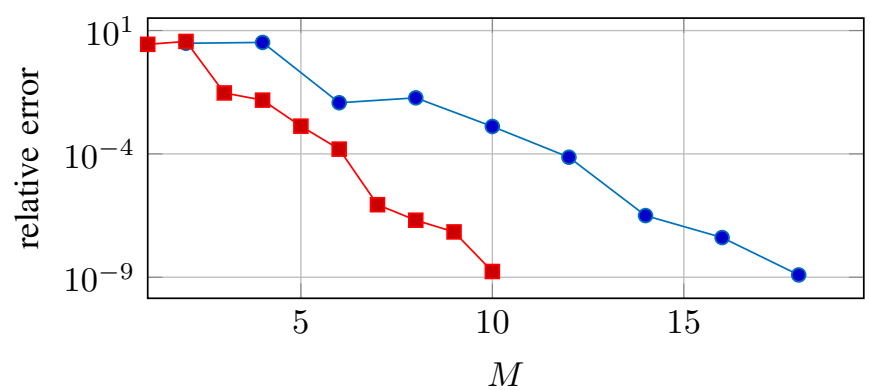

Fig. 4. Greedy frequency algorithm - convergence rate of greedy algorithms

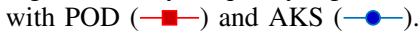
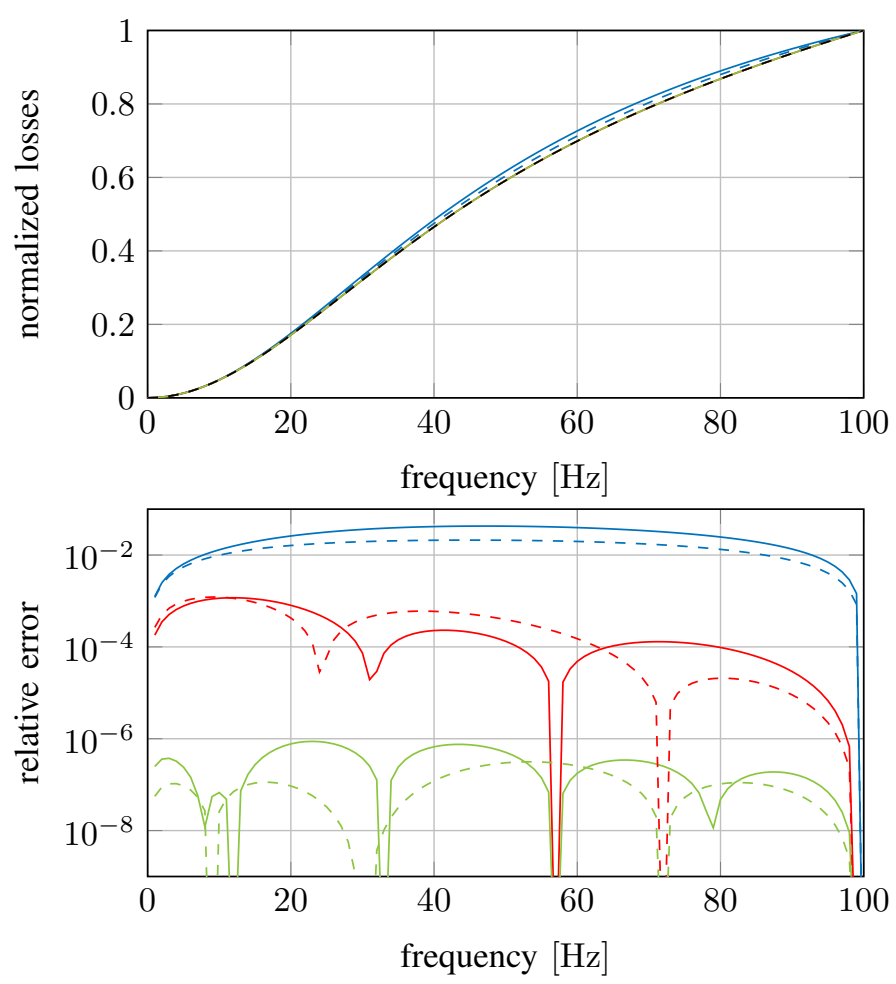

Fig. 5. Greedy frequency algorithm - normalized losses in transformer core with POD and AKS (up) and $L^{2}$-relative error (down). FEM (- - ), POD $\mathrm{M}=2(\square), 4(-), 6(-)$, AKS $\mathrm{M}=4(---), 8(---), 12(---)$

what amounts to twice the size of the POD for the same number of frequencies.

In Fig. 5, the frequency response of the core losses is depicted. The time evolution is given in Fig. 6 as well. Those results are very similar to the ones obtained via the uniform selection process because this test case does not present high resonances as described in [13]. Therefore a uniform scanning over the frequency range is sufficient to provide a well-defined overall response in the frequency domain. The selected frequencies are hierarchical since the algorithms are greedy: $[0,100,57,33,12,11,23,72,74] \mathrm{Hz}$ (POD) and $[0,100,99,72,31,9,85,28,5] \mathrm{Hz}(\mathrm{AKS})$ in the order of selection.

One can see that the correct evaluation of the global quantity
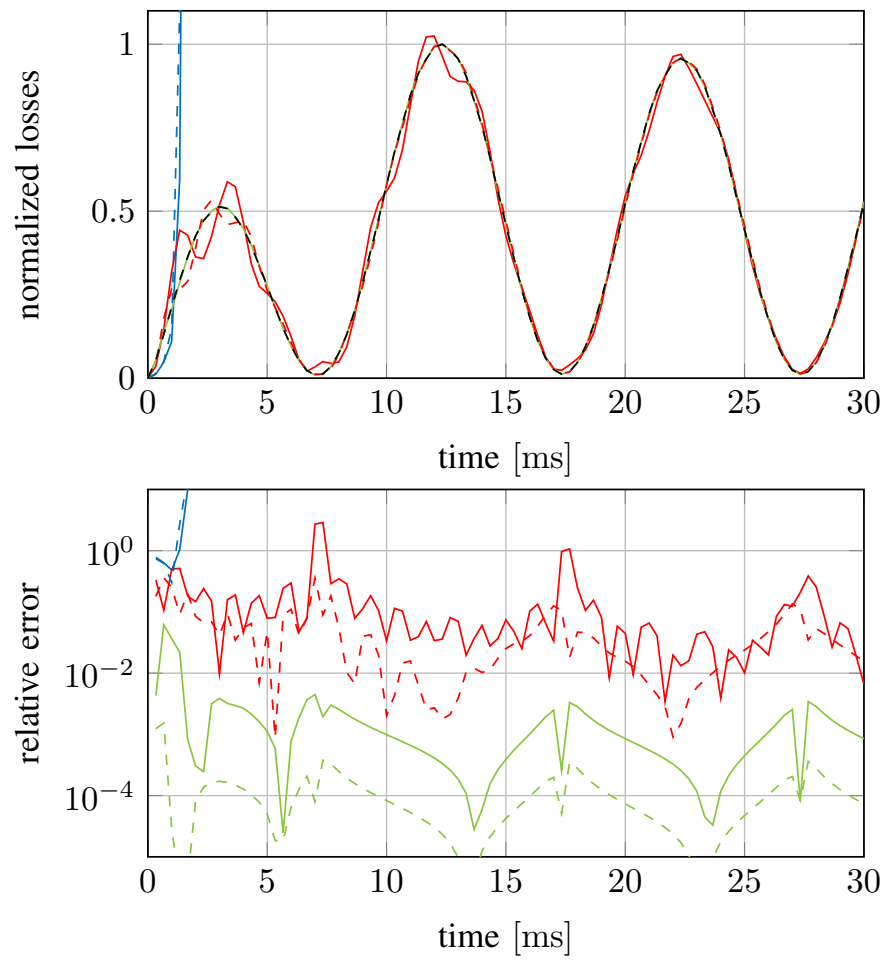

Fig. 6. Greedy frequency algorithm - normalized losses in transformer core with POD and AKS (up) and $L^{2}$-relative error (down). FEM (-- ), POD $\mathrm{M}=2(-), 4(-), 6(-), \mathrm{AKS} \mathrm{M}=4(---), 8(---), 12(---)$

in frequency allows the corresponding projector operator to precisely describe the transient of the transformer.

\section{B. Projection operator $\Psi$ generated in time}

Unlike [12], where the projection operator $\Psi$ is constructed from the $K_{s}$ first time steps, we apply the above described algorithm together with the POD. It allows to automatically determine the projector operator. The results are shown in Fig. 7: by considering too small bases, based on the first time steps, the solution clearly diverges from the reference solution, e.g. $M=2$ or 4 . By increasing the number of trials $n b$ required to consider the reduced basis exact, the time error evolution is lowered, e.g. $M=5$, 6 . With $M=10$, the number of predictions is sufficiently high to obtained an average $L^{2}$ error around $1 \%$. Similarly, good convergence behaviour is observed for a local quantity, e.g. the magnetic flux density $b$, the absolute error of which is plotted in Fig. 8 at instant $25 \mathrm{~ms}$ when the reference model has not been computed during the greedy algorithm (it stops before $10 \mathrm{~ms}$ ).

\section{CONCLUSION}

In this paper, we have compared the performance of POD and AKS methods in the time domain, considering both local and global quantities. Two approaches has been investigated: the generation of the projection operator in frequency domain and in time domain with original greedy algorithms avoiding any human arbitrary decision. With the so-selected snapshots both local and global quantities are accurately computed. 

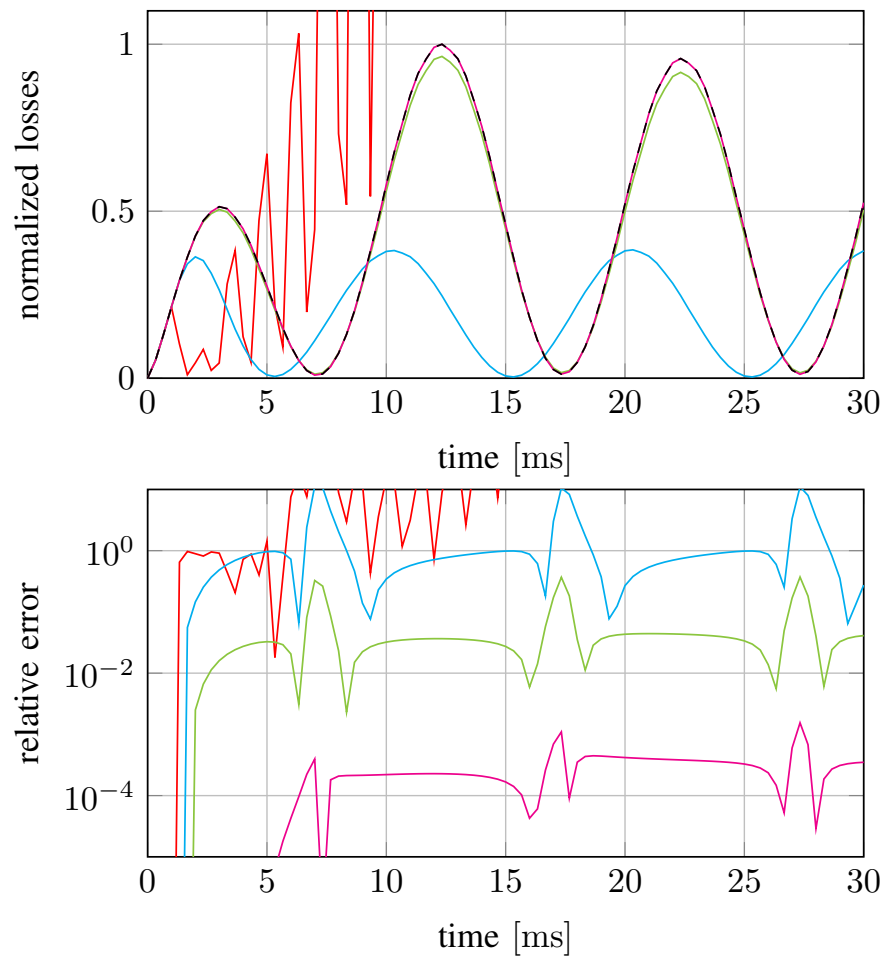

Fig. 7. Greedy time algorithm - normalized losses in the transformer core (up) and $L^{2}$-relative error (down). FEM (-- ), POD M=4( - ), 5( - ), $6(-), 10(-)$

Those approaches have been applied to a linear isotropic conductive transformer presenting a smooth behaviour. This test case showed the easiness to compute a reduced order model of size 10 compared to an original 4175-unknown system-allowing a reduction of $99.7 \%$. Further work concerns the inclusion of stronger eddy current effects, saturation as well as a parametric load-dependence.

\section{REFERENCES}

[1] B. Gustavsen, "Wide Band Modeling of Power Transformers," IEEE Trans. Power Delivery, vol. 19, no. 1, pp. 414-422, 2004.

[2] E. Amoiralis, M. Tsili, A. G. Kladas, "Transformer design and optimization: a literature survey," IEEE Trans. Power Delivery, vol. 24, no. 4, pp. 1999-2024, 2009.

[3] J. Chen, Z. Chen, T. Cui, L.-B. Zhang, "An adaptive finite element method for the eddy current model with circuit/field couplings," SIAM Journal on Scientific Computing, vol. 32, no. 2, pp. 1020-1042, 2010.

[4] Z. Liu, S. Liu, and O. A. Mohammed, "A Practical Method for Building the FE-Based Phase Variable Model of Single Phase Transformers for Dynamic Simulations," IEEE Trans. Magn., vol. 43, no. 4, pp. 17611764, 2007.

[5] T. S. Nguyen, J.-M. Guichon, O. Chadebec, G. Meunier, "Equivalent circuit synthesis method for reduced order models of large scale inductive PEEC circuits," In Proceedings of the 2012 15th Biennial IEEE Conference on Electromagnetic Field Computation (CEFC), Oita, Japan, Nov. 11-14, 2012.

[6] J. Gyselinck, R. V. Sabariego, "Airgap reluctance identification for the magnetic equivalent circuit modelling of induction machines," In Proceedings of the Conference on the Computation of Electromagnetic Fields, COMPUMAG 2013, July 2013, Budapest, Hungary.

[7] H. Allag, A. Kedous-Lebouc, M. E. H. Latreche, "Preisach hysteresis implementation in reluctance network method, comparison with finite element method," In Proceedings of the XX Symposium Electromagnetic
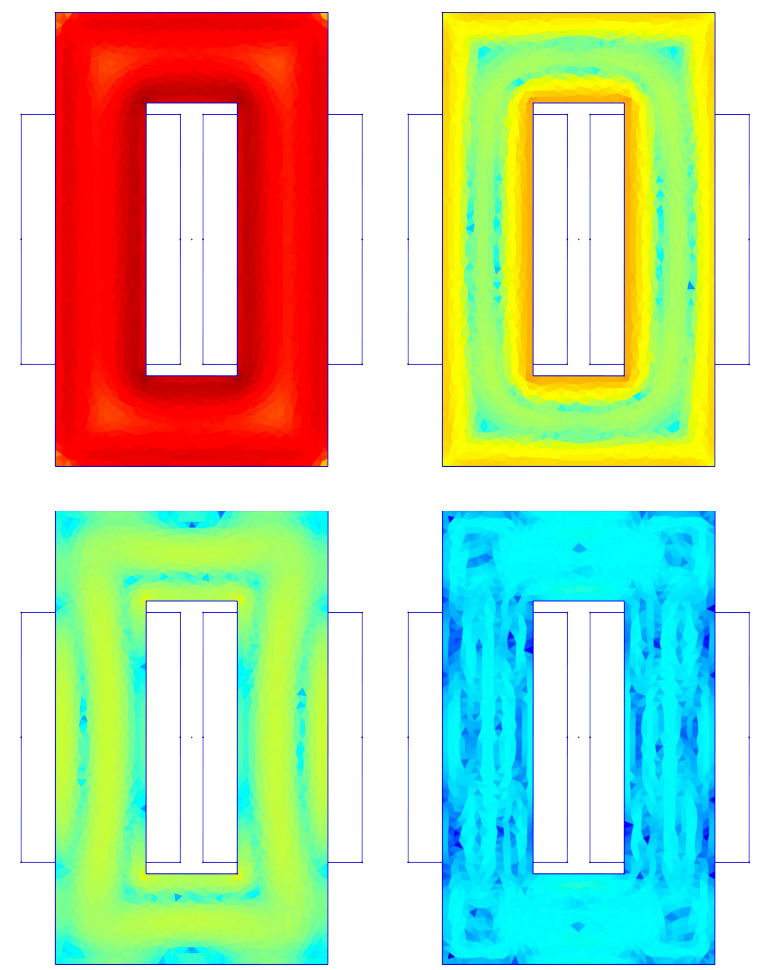

Fig. 8. Absolute error of $b$ for $M=4,5,6,10$ at $25 \mathrm{~ms}$ (from up-left to down-right). Logarithmic scale range: $10^{-6}$ (dark blue), $10^{-3}$ (yellow) and $10^{1}$ (dark red).

Phenomena in Nonlinear Phenomena, EPNC 2008, July 2008, Lille, France.

[8] W. Schilders, H. V. der Vorst, J. Rommes, Model order reduction: theory, research aspects and applications, Springer-Verlag, 2008.

[9] Y. Sato, H. Igarashi, "Model reduction of three-dimensional eddy current problems based on the method of snapshots," IEEE Trans. Magn., vol. 49, no. 5, pp. $16971700,2013$.

[10] A. Chatterjee, "An introduction to the proper orthogonal decomposition," Current science, vol. 78, no. 7, pp. 808-817, 2000.

[11] Z. Bai, "Krylov subspace techniques for reduced-order modeling of large-scale dynamical systems," Applied Numerical Mathematics, vol. 43, no. 1 , pp. 9-44, 2002.

[12] A. Pierquin, T. Henneron, S. Clénet, S. Brisset, "Model Order Reduction of Magnetoquasistatic Problems Based on POD and Arnoldi-based Krylov Methods," IEEE Trans. Magn., vol.51, no. 3, pp. 7206204-1-4, 2015.

[13] Y. Paquay, C. Geuzaine, MD R. Hasan, R. V. Sabariego, "Reduced Order Model for Accounting for High Frequency Effects in Power Electronic Components," IEEE Trans. Magn., vol.52, no. 3, 2016.

[14] A. Odabasioglu, M. Celik, L. T. Pileggi,"Prima: passive reducedorder interconnect macromodeling algorithm," In Computer-Aided Design, 1997. Digest of Technical Papers., 1997 IEEE/ACM International Conference, pp. 58-65, Nov. 1997.

[15] A. Buffa, Y. Maday, A. T. Patera, C. Prudhomme, G. Turinici, "A priori convergence of the Greedy algorithm for the parametrized reduced basis method," ESAIM: Mathematical modelling and numerical analysis, vol. 46, no. 3, pp. 595-603, 2012.

[16] A. Paul-Dubois-Taine, D. Amsallem, "An adaptive and efficient greedy procedure for the optimal training of parametric reduced-order models," Int. J. Numer. Meth. Engng., vol. 102, no. 5, pp. 1262-1292, 2015.

[17] T. Henneron, S. Clenet, "Model Order Reduction of Non-Linear Magnetostatic Problems Based on POD and DEI Methods," IEEE Trans. Magn., vol.50, no. 2, pp. 33-36, 2014.

[18] T. Henneron, S. Clenet, "Model-Order Reduction of Multiple-Input NonLinear Systems Based on POD and DEI Methods," IEEE Trans. Magn., vol.51, no. 3, pp. 1-4, 2015. 